\title{
Start des Weaning-Registers - jetzt schon eine Erfolgsgeschichte
}

\author{
Start of the Weaning-Database - Already a Success Story
}

Autoren

Institute

\author{
B. Schönhofer ${ }^{1}$, H. Teschler ${ }^{2}$
}

KRH Klinikum Oststadt-Heidehaus, Abt. Pneumologie und intern. Intensivmedizin

2 Ruhrlandklinik, Essen

\section{Bibliografie}

Dol $10.1055 / \mathrm{s}-0028-1119557$

Pneumologie 2009; 63: 164-165

(c) Georg Thieme Verlag KG

Stuttgart · New York

ISSN 0934-8387

Korrespondenzadresse

Prof. Dr. med.

Bernd Schönhofer

KRH Klinikum Oststadt-

Heidehaus

Abt. Pneumologie

u. intern. Intensivmedizin

Podbielskistr. 380

30659 Hannover

bernd.schoenhofer@t-online.de
Bereits der Name „Deutsche Gesellschaft für Pneumologie und Beatmungsmedizin“ bringt den Stellenwert der Beatmungsmedizin in der DGP unmissverständlich zum Ausdruck. Die wachsende Bedeutung der Beatmungsmedizin wird auch durch die Versorgungsrealität unterstrichen: In den vergangenen 15-20 Jahren haben sich immer mehr pneumologische Kliniken und Abteilungen auf die schwierige Entwöhnung vom Respirator (Weaning) spezialisiert.

Die DGP sieht es als ihre Aufgabe an, die Qualität dieser Weaningzentren zu sichern. $\mathrm{Zu}$ diesem Zweck wurde im Jahr 2007 eine elfköpfige Arbeitsgruppe „Weaningzentren“ gegründet. Die Aktivitäten der Arbeitsgruppe konzentrierten sich auf die Entwicklung eines Registers der Weaningpatienten und auf das Akkreditierungsverfahren für Weaningzentren. An dieser Stelle möchten wir den Experten der AG ausdrücklich danken für ihre große Unterstützung.

Nach einem Jahr Entwicklungszeit und zwei aufwendigen Testphasen mit den in der AG vertretenen Weaningzentren nahm das Weaning-Register im Januar 2009 seinen regulären Betrieb auf. Schon wenige Tage nach dem Start haben sich mehr als 20 Weaningzentren zur Teilnahme registrieren lassen.

Das Weaning-Register erfasst Patienten, die nach mindestens 7 Tagen Langzeitbeatmung schwierig zu entwöhnen sind und/oder zur Respiratorentwöhnung in ein spezielles Weaningzentrum verlegt wurden. Entsprechend der Erhebung der DGP aus dem Jahr 2007 betrifft dies mindestens 3000 Patienten jährlich. Der Datensatz umfasst anamnestische und diagnostische Daten, Angaben zu Dauer und Verlauf der Beatmung, zum Prozedere der Entwöhnung, Daten zu aufgetretenen Komplikationen und zum Outcome der Patienten. Hohe Priorität legte die Arbeitsgruppe auf eine schlanke Datensatzdefinition durch Beschränkung auf medizinisch zentrale, klinisch aussagekräftige Informationen.
Die Arbeitsgruppe „Weaningzentren“ hat während der Entwicklungsphase des Registers eng mit dem Institut für Lungenforschung (ILF) unter der Projektleitung von Frau Dr. Nina Hämäläinen kooperiert. Die Programmierung der Datenbank und der Internet-basierten Benutzerschnittstelle lag in den Händen des ILF, das auch den Betrieb der Datenbank und die Benutzerverwaltung übernimmt.

Die Oberfläche zur Dateneingabe unterstützt den Anwender durch interaktive Hilfen. Da es sich um ein Serverkonzept handelt, muss keine besondere Software installiert werden; benötigt werden lediglich ein Internetzugang, ein üblicher Internet-Browser und die individuelle, zentrumsspezifische Zugangsberechtigung.

Parallel zu der überzeugend gelungenen technischen Umsetzung genehmigte die zuständige Aufsichtsbehörde das vom ILF entwickelte Verfahren zur Pseudonymisierung der Daten. Für die Patientenaufklärung und Einwilligung stellt das ILF allen Zentren die notwendigen Unterlagen zur Verfügung.

Besondere Bedeutung hat das Weaning-Register im Zusammenhang mit dem zur Zeit in der Entwicklung befindlichen Akkreditierungsprozess pneumologischer Weaningzentren. Die Teilnahme am elektronischen Patientenregister und die Dokumentation von mindestens 40 Weaningpatienten in diesem System ist obligatorische Voraussetzung für die Akkreditierung. Mit dem Erwerb der Akkreditierung ist die kontinuierliche Teilnahme des Weaningzentrums am Register verpflichtend.

Der Kriterienkatalog zur Akkreditierung wird derzeit vorbereitet. Die AG Weaningzentren arbeitet daran in enger Absprache mit dem Vorstand der DGP. Im Frühjahr 2009 werden die Kriterien und Verfahrensregeln zur Akkreditierung in der Zeitschrift „Pneumologie“, durch EmailVersand und auf der Webseite der DGP veröffentlicht. Von diesem Zeitpunkt an steht interessier- 
ten Zentren auch die Anmeldung zum Akkreditierungsverfahren offen.

Die Akkreditierung und Einschreibung im Weaning-Register bilden fortan die Grundlage für eine bundesweite Qualitätssicherung der Weaningzentren unter der Schirmherrschaft der DGP. Zentrumsspezifische Auswertungen als Basis für die interne Qualitätsplanung werden allen teilnehmenden Zentren zur Verfügung gestellt. Welche Optionen der Auswertung konkret möglich sind, hängt von mehreren bisher unbekannten Größen ab, z. B. der Anzahl teilnehmender Zentren, der Eingabequalität etc. Wir hoffen, im Herbst/Winter 2009 bei einer gemeinsamen Veranstaltung der teilnehmenden Zentren erste Übersichtsdaten präsentieren und gewünschte Auswertungen diskutieren zu können.

Mittelfristig wird das Weaning-Register für Kostenträger und Politik verlässliche Zahlen zur Versorgungssituation der Beatmungsentwöhnung in Deutschland liefern.
Der Antrag auf Teilnahme am Weaning-Register ist durch das Zentrum formlos an das ILF zu richten. Die Adresse lautet:

Institut für Lungenforschung e.V.

Frau Dr. Nina Hämäläinen

Robert-Koch-Allee 23

82131 Gauting

Wir hoffen auf ein reges Interesse am Weaning-Register

Prof. Dr. Bernd Schönhofer

Federführung der

Prof. Dr. Helmut Teschler

AG Weaningzentren
Präsident der DGP 